\title{
Topical application of adipose tissue- derived mesenchymal stem cells (ADMSCs) reduced cerebral edema in experimental traumatic brain injury (TBI) - a preliminary study
}

Hui Ma ${ }^{1+}$, Lian Xu Cui ${ }^{2 \dagger}$, Ping Kuen Lam¹, Cindy S. W. Tong ${ }^{1}$, Kin K. Y. Lo ${ }^{1}$, George K. C. Wong ${ }^{1}$ and Wai Sang Poon ${ }^{*}$

\begin{abstract}
Background: Our previous studies showed that topical application of mesenchymal stem cells (MSCs) improved functional recovery in rat traumatic brain injury (TBI) model, and hypoxic precondition further enhanced the therapeutic effects of MSCs. There was no previous study on the attenuation of cerebral edema by MSCs. We investigated whether topical application of normoxic and hypoxic MSCs could reduce cerebral edema in an experimental TBI model.

Methods: Two million normoxic $(N=24)$ and hypoxic $(N=24)$ MSCs were applied topically to exposed the cerebral cortex in a controlled cortical impact (CCl) model. The MSCs were fixed in position with fibrin glue. No treatment was given to control animals (TBI only: $n=24)$. After surgery, four animals in each group were sacrificed daily (day 1 to day 6) for edema evaluation. Normal animals without TBI were used as reference $(n=4)$. The expressions of GFAP, AQP4, and MMP9 were also investigated by immunofluorescence staining and RT-PCR at day 3.

Results: The edema peaked within 3 days after TBI. Compared with the control, hypoxic MSCs reduced brain water content significantly $(p<0.05)$. Both hypoxic and normoxic MSCs downregulated the expression of MMP9 and normalized AQP4 distribution to astrocyte end feet.
\end{abstract}

Conclusion: Our preliminary study showed that topical application of hypoxic MSCs suppressed both vasogenic and cytotoxic edema formation.

Keywords: Topical, MSCs, Cerebral edema, TBI

\footnotetext{
* Correspondence: wpoon@surgery.cuhk.edu.hk

${ }^{\dagger} \mathrm{Hui} \mathrm{Ma}$ and Lian Xu Cui are co-first authors of the study.

'Division of Neurosurgery, Chow Tai Fook-Cheng Yu Tung Surgical Stem Cell

Research Center, Department of Surgery, The Chinese University of Hong

Kong, Shatin, Hong Kong, SAR, China

Full list of author information is available at the end of the article
}

(c) The Author(s). 2021 Open Access This article is licensed under a Creative Commons Attribution 4.0 International License, which permits use, sharing, adaptation, distribution and reproduction in any medium or format, as long as you give appropriate credit to the original author(s) and the source, provide a link to the Creative Commons licence, and indicate if changes were made. The images or other third party material in this article are included in the article's Creative Commons licence, unless indicated otherwise in a credit line to the material. If material is not included in the article's Creative Commons licence and your intended use is not permitted by statutory regulation or exceeds the permitted use, you will need to obtain permission directly from the copyright holder. To view a copy of this licence, visit http://creativecommons.org/licenses/by/4.0/. The Creative Commons Public Domain Dedication waiver (http://creativecommons.org/publicdomain/zero/1.0/) applies to the data made available in this article, unless otherwise stated in a credit line to the data. 


\section{Background}

Brain edema was defined as "an expansion of brain volume which increases intracranial pressure', impairs cerebral perfusion, and causes additional ischemic injuries" [1]. For decades, edema has been a target in the treatment of traumatic brain injury. Conventional managements to reduce cerebral edema include (i) general measures: optimal head and neck position, adequate oxygenation, maintenance of normotension, management of fever and hyperglycemia, and nutritional support; (ii) specific interventions: controlled hyperventilation, osmotherapy (mannitol, hypertonic saline), corticosteroid administration, and pharmacological coma (barbiturates); and (iii) surgical procedures: cerebrospinal fluid (CSF) drainage, decompressive craniectomy, and decompressive laparotomy [2]. However, these passive treatments can induce complications such as renal failure and acute respiratory distress syndrome [2]. Classically, there are two types of edema associated with traumatic brain injury, namely vasogenic edema and cytotoxic/cell edema [3, 4]. Vasogenic edema is caused by disruption of blood-brain barrier (BBB) after TBI, resulting in water movement from the blood vessels to extracellular space and an increase in brain water content. Cytotoxic edema, also known as cellular or ionic edema, is defined as "cellular failure with disrupted ionic pump with anaerobic metabolism" which is caused by ischemia after TBI [5].

Mesenchymal stem cells (MSCs) are able to selfrenewal and differentiate into multiple cell lineages. To accelerate functional recovery, MSCs bio-modulate the local microenvironment, suppress inflammatory response, and promote angiogenesis and regeneration in addition to the trans-differentiation after transplantation [6]. Our recent study showed that hypoxic preconditioning of MSCs enhanced tissue repair and functional recovery in experimental TBI [7]. To our knowledge, there are no reports on the attenuation of cerebral edema by MSCs.

We would like to investigate whether topical application of MSCs could reduce cerebral edema in a rodent model of TBI.

\section{Methods}

MSCs were derived from the subcutaneous adipose tissue of male SD rats. Cultured under either normoxic $\left(18 \% \mathrm{O}_{2}\right)$ or hypoxic $\left(5 \% \mathrm{O}_{2}\right)$, all MSCs expressed CD29 and CD90 but not CD45. The MSCs had adipogenic, chondrogenic, and osteogenic differentiation potentials in vitro under specific culture conditions. Female SD rats, aging 10-12 weeks were used for the study (Laboratory Animal Services Center, CUHK). Traumatic brain injury was induced by controlled cortical impact (CCI) [7] (Fig. 1). After surgery, 2 million normoxic $(n=24)$ and hypoxic $(n=24)$ MSCs were applied topically to the exposed brain cortex. A thin layer of fibrin glue was used to keep cells in position. No treatment was given to animals in the control group $(n=24)$ (Table 1$)$.

Four animals in each group were sacrificed daily until day 6, and normal animals were used as reference $(n=$ 4). For each animal, the injured hemisphere was collected and dried in the oven for $36 \mathrm{~h}$. Wet weight and dry weight were measured respectively. Brain water content was calculated according to the formula: (wet weight-dry weight)/wet weight $\%$.

Hydrated paraffin tissue slices were stained by antiGFAP (Abcam, Cambridge, UK) (a marker of astrocytes) and anti-AQP4 (Abcam, Cambridge, UK) (a marker of water channel on astrocytes) antibody. Real-time PCR was used for the determination of MMP9 (Abcam, Cambridge, UK) (marker of vasogenic edema) expression.

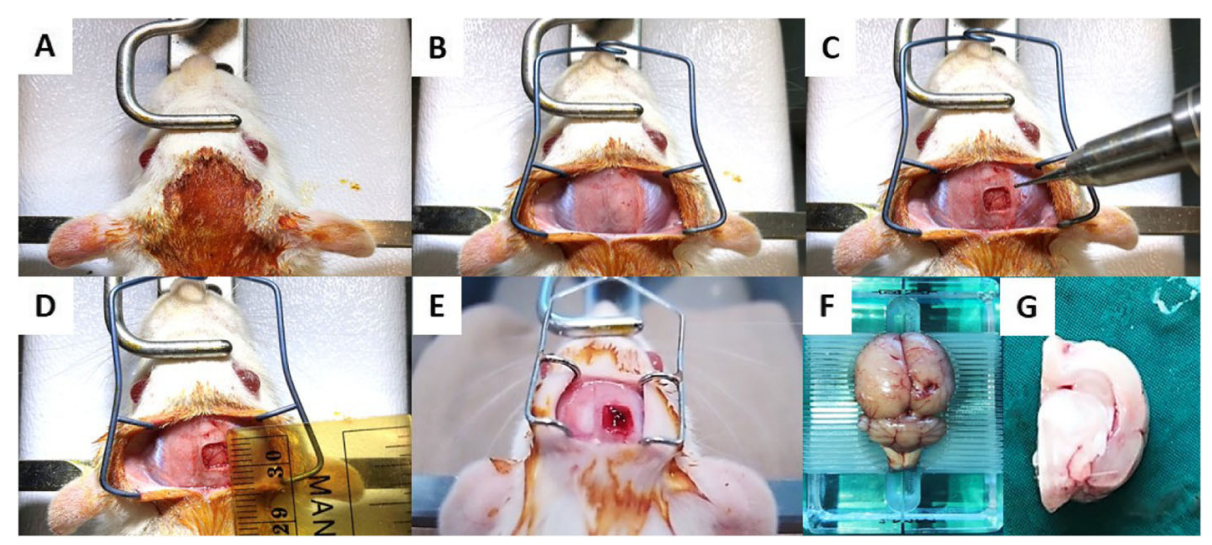

Fig. 1 The process of controlled cortical injury $(\mathrm{CCl})$ and injured hemisphere collection. a-e Process of CCI model on SD rat. $\mathbf{f}$ Right hemisphere was injured. $\mathbf{g}$ The hemisphere harvested for brain water content measurement 
Table 1 Animal groupings in the study

\begin{tabular}{lll}
\hline Groupings & Number of animals & Treatment \\
\hline Control & 24 & TBI without treatment \\
MSCS & 24 & TBI +2 million MSCs \\
Hypoxic MSCs & 24 & TBI +2 million hypoxic MSCs \\
\hline
\end{tabular}

\section{Results}

\section{Hypoxic MSCs reduced brain water content}

The brain water content increased remarkably within 3 days after TBI (Fig. 2) (Table 2). Then, it reduced significantly in the hypoxic MSC group ( $p<0.05$ vs control), whereas no significant change was found in the normoxic group (Fig. 3).

\section{Hypoxic MSCs normalized the AQP4 distribution}

Double fluorescent staining of GFAP and AQP4 indicated that AQP4 distribution was normalized to the end feet of astrocytes after either normoxic or hypoxic MSC treatment, whereas a positive fluorescent signal was observed around the cell body of astrocyte in the control group (Fig. 4).

\section{Hypoxic MSCs downregulated the expression of MMP-9}

Expression of MMP9 was downregulated significantly in both normoxic ( $p<0.05$ vs control) and hypoxic $(p<$ 0.05 vs control) groups on day 3 . No significant difference was found between two different MSC groups (Fig. 5) (Table 3).

\section{Discussion}

The pathophysiology of brain edema involves vasogenic and cytotoxic cascades. Vasogenic edema is associated with blood-brain barrier (BBB) integrity. Also, brain water content increases due to BBB dysfunction. In this study, we demonstrated that topical application of hypoxic MSCs reduced brain water content significantly on day 3. This effect was not observed in the normoxic MSC treatment group. Previous study showed hypoxic preconditioning enhanced therapeutic effects of MSCs [7]. Thus, it was believed that the suppression effects against cerebral edema were stronger in the hypoxic MSC group. Animal studies showed the upregulation of MMP9 in TBI [8, 9]. MMP9 degrades extracellular matrix proteins, including neurovascular basal lamina and tight junction proteins of BBB [10]. The downregulation of MMP9 by MSC treatment could reduce edema formation and maintain $\mathrm{BBB}$ integrity [10]. AQP4, one of the water channel proteins, is a key factor in the development and resolution of cerebral edema [11]. Normally, it locates in the perivascular astrocyte end feet [12]. After traumatic brain injury occurs, astrocytosis is activated, and the AQP4 signal is not limited to the end feet. It is also detected all reactive astrocytes [12]. Topical application of MSCs suppressed neuro-inflammation

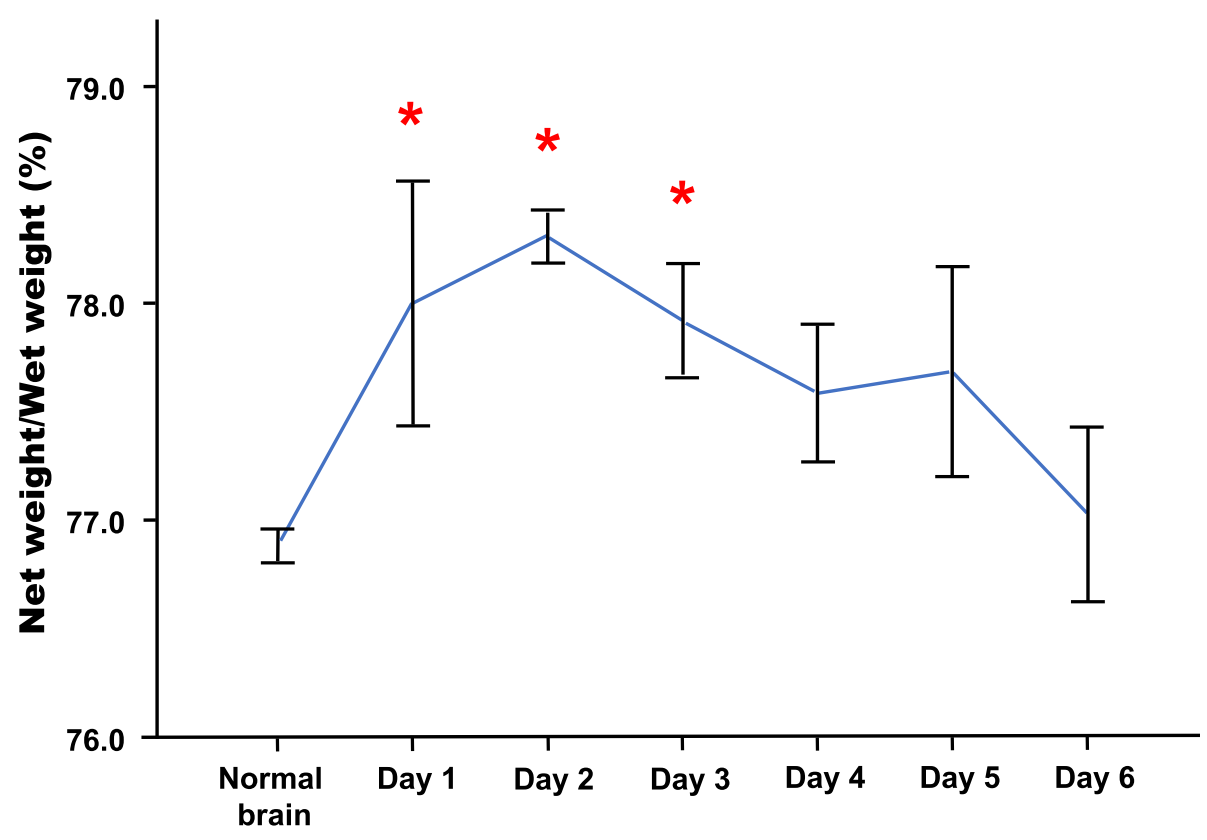

Fig. 24 animals were sacrificed daily until day 6 after TBI. The injured hemisphere was harvested and measure brain water content. Compared with hemisphere from normal animals, the brain water content was significantly higher from day 1 to day $3\left({ }^{*} p<0.05\right.$ vs control) (net weight $=$ wet weight-dry weight) 
Table 2 The average wet and dry weight of the injured hemisphere

\begin{tabular}{llll}
\hline Groups/time point & Wet weight $(\mathbf{g})$ & Dry weight $(\mathbf{g})$ & Water content (\%) \\
\hline Normal & 0.73 & 0.17 & 76.88 \\
Control-D1 & 0.73 & 0.16 & $78.00^{*}$ \\
Control-D2 & 0.68 & 0.15 & $78.31^{*}$ \\
Control-D3 & 0.70 & 0.15 & $77.92^{*}$ \\
Control-D4 & 0.64 & 0.14 & 77.59 \\
Control-D5 & 0.67 & 0.15 & 77.69 \\
Control-D6 & 0.67 & 0.15 & 77.03 \\
MSCs-D3 & 0.63 & 0.14 & 77.90 \\
Hypoxic MSCs-D3 & 0.70 & 0.16 & $76.95^{\#}$ \\
\hline
\end{tabular}

${ }^{*} p<0.05$ vs normal

${ }^{\#} p<0.05$ vs control

by triggering reactive astrocytosis at the early phase [6]. In this study, topical MSCs normalized AQP4 distribution in astrocytes. In the tissue without MSC treatment, the astrocytes underwent swelling after TBI, resulting in the opening and re-location of the water channel on the cell body. The topically applied MSCs contributed to the suppression of cerebral edema and the reduction of astrocytes swollen, shown as the normalization of the AQP4 distribution. Although the underlying mechanism is not yet clear, there are two hypotheses to explain the attenuation of cerebral edema. Firstly, numerous studies have shown that the transplanted MSCs were capable to express a marker of astrocytes (GFAP) in vivo, which may help maintain BBB integrity [13]. Secondly,
MSCs secrete soluble factors including growth factors, cytokines, and chemokines, through paracrine activity [13]; they might biomodulate the neuro-inflammation associated with cerebral edema.

However, we have some limitations in this study. First, we did not measure brain water content at the acute phase after TBI, such as $1 \mathrm{~h}, 6 \mathrm{~h}$, and $12 \mathrm{~h}$. Second, we did not compare therapeutic effects of normoxic and hypoxic MSCs in the acute phase. Third, we compare water content only in the injured hemisphere instead of the whole brain.

\section{Conclusion}

In this study, we demonstrated topical application could deliver a large amount of MSCs safely and effectively to

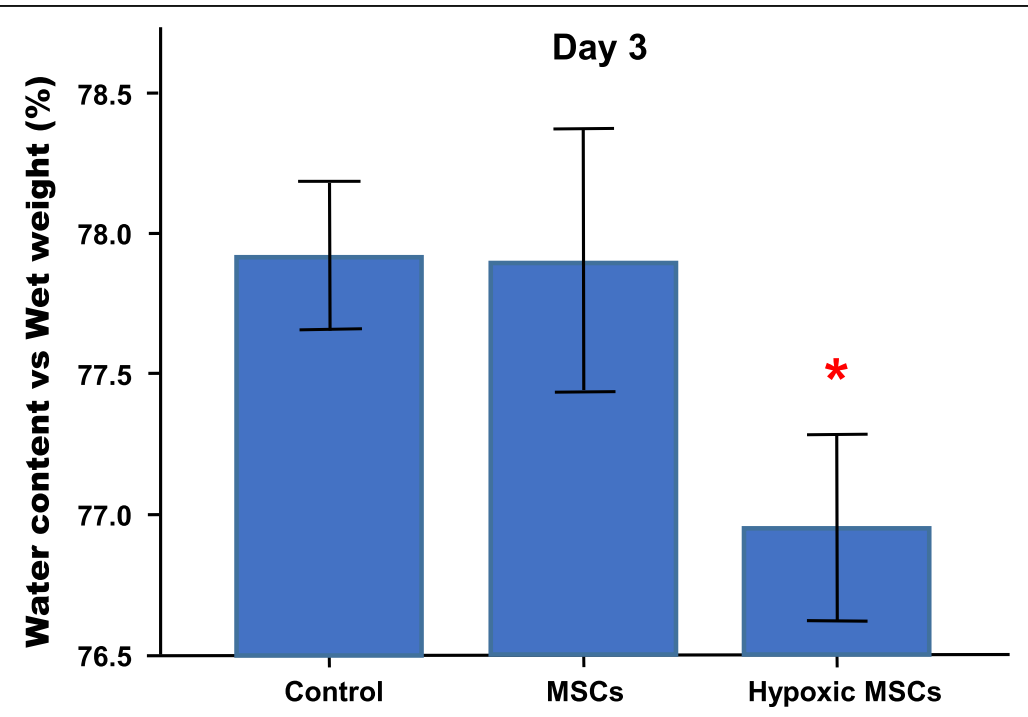

Fig. 3 The injured hemispheres were harvested from animals in different groups on day 3. The hypoxic MSC-treated brain showed lower water content than control ( ${ }^{*} p<0.05$ vs control), and no remarkable change was found between normoxic MSC-treated brain and control 


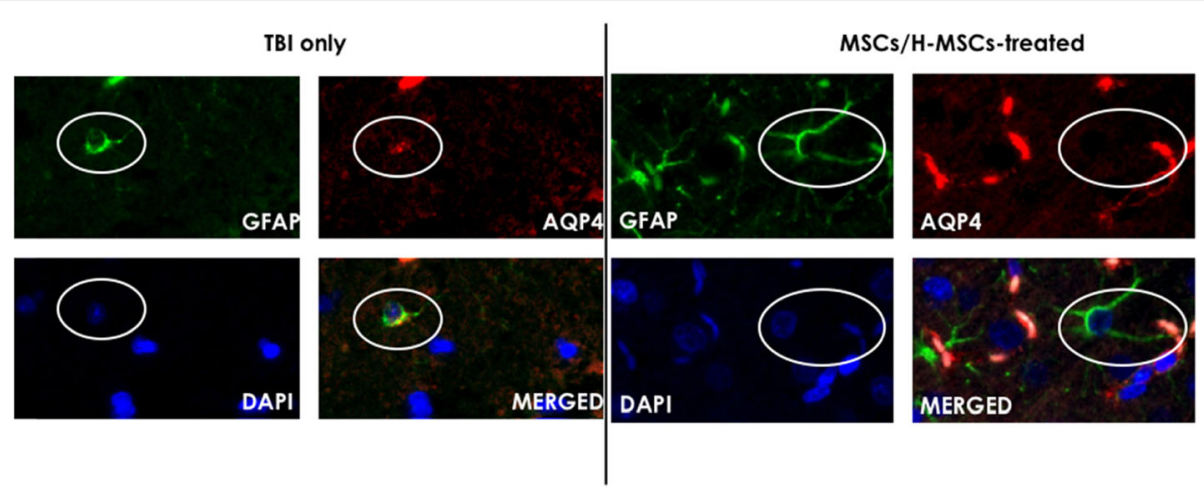

Fig. 4 Picture of double immune-florescent staining of GFAP and AQP4. After TBI, distribution of AQP4 signal spread to cell body of astrocytes. With treatment of normoxic/hypoxic MSCs, the signal located to end feet of astrocytes

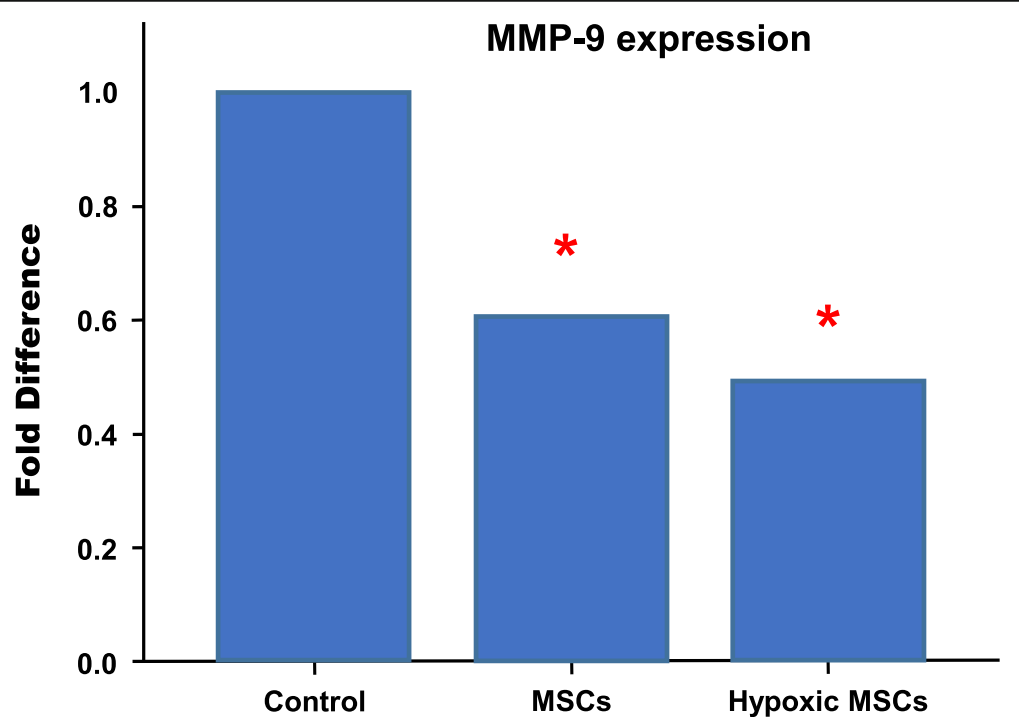

Fig. 5 RT-PCR results indicated MMP9 expression was downregulated significantly after both normoxic and hypoxic MSC transplantation ${ }^{*} p<$ 0.05 vs control). No statistical difference was found between two cell treatments 
Table 3 List of primers used in RT-PCR

\begin{tabular}{ll}
\hline GAPDH & Forward AGA CAG CCG CAT CTT GT \\
& Reverse CTT GCC GTG GGT AGA GTC AT \\
MMP-9 & Forward CCC CAC TTA CTT TGG AAA CGC \\
& Reverse ACC CAC GAC GAT ACA GAT GCTG
\end{tabular}

the injured brain. Hypoxic MSCs reduced brain water content 3 days after the injury happened, both normoxic and hypoxic MSC treatment downregulated provasogenic edema gene expression and normalize water channel distribution in astrocytes.

\section{Abbreviations}

ADMSCs: Adipose tissue-derived mesenchymal stem cells; BBB: Blood-brain barrier; CCl: Controlled cortical impact; CSF: Cerebrospinal fluid;

MSCs: Mesenchymal stem cells; TBI: Traumatic brain injury

\section{Acknowledgements}

None.

\section{Authors' contributions}

Hui MA performed the experiments and wrote the manuscript. C.S.W. Tong and K.K. Lo helped with the immunohistochemistry staining. Lian Cui and PK designed the study. PK Lam, George Wong, and WS Poon supervised the study, provided language help, and writing assistance. All authors reviewed and commented on the manuscript. The authors approved the final version of the manuscript.

\section{Funding}

This research did not receive any specific grant from funding agencies in the public, commercial, or not-for-profit sectors.

\section{Availability of data and materials}

Please contact the corresponding author if any data or materials are required.

\section{Ethics approval and consent to participate}

All animal procedures were conducted in accordance with the guidelines in the Animals (Control of Experiments) Ordinance (Chapter 340), Department of Health, Hong Kong, and the study was approved by the Animal Experimentation Ethics Committee of The Chinese University of Hong Kong.

\section{Consent for publication}

Not applicable.

\section{Competing interests}

There is no conflict of interest in this research.

\section{Author details}

'Division of Neurosurgery, Chow Tai Fook-Cheng Yu Tung Surgical Stem Cell Research Center, Department of Surgery, The Chinese University of Hong Kong, Shatin, Hong Kong, SAR, China. ${ }^{2}$ Division of Neurosurgery, Department of Surgery, Fo Shan First People's Hospital, Foshan, Guangdong, China.

Received: 4 May 2020 Accepted: 1 December 2020

Published online: 04 January 2021

\section{References}

1. Unterberg AW, Stover J, Kress B, Kiening KL. Edema and brain trauma. Neurosci. 2004;129:1019-27.

2. Gonda DD, Meltzer HS, Crawford JR, Hilfiker ML, Shellington DK, Peterson BM, Levy ML. Complications associated with prolonged hypertonic saline therapy in children with elevated intracranial pressure. Pediatr Crit Care Med. 2013;14:610-20.

3. Klatzo I. Pathophysiological aspects of brain edema. Acta Neuropathol. 1987; 72:236-9.
4. Donkin JJ, Vink R. Mechanisms of cerebral edema in traumatic brain injury: therapeutic developments. Curr Opin Neurol. 2010;23:293-9.

5. Mahajan S, Hemant B. Cerebral oedema: pathophysiological mechanisms and experimental therapies. J Neuroanaesthesiol Crit Care. 2016;3:22.

6. Lam PK, Lo AW, Wang KK, Lau HC, Leung KK, Li KT, Lai PB, Poon WS. Transplantation of mesenchymal stem cells to the brain by topical application in an experimental traumatic brain injury model. J Clin Neurosci. 2013:20:306-9.

7. Ma H, Lam PK, Tong CSW, Lo KKY, Wong GKC, Poon WS. The neuroprotection of hypoxic adipose tissue-derived mesenchymal stem cells in experimental traumatic brain injury. Cell Transplant. 2019;28(7):874-84.

8. Shigemori Y, Katayama Y, Mori T, Kawamata T. Matrix metalloproteinase-9 is associated with blood-brain barrier opening and brain edema formation after cortical contusion in rats. Acta Neurochir Suppl. 2006;96:130-3.

9. Hayashi T, Kaneko Y, Yu S, Bae E, Stahl CE, Kawase T, van Loveren H, et al. Quantitative analyses of matrix metalloproteinase activity after traumatic brain injury in adult rats. Brain Res. 2009;1280:172-7.

10. Rosenberg GA. Matrix metalloproteinases in neuroinflammation. Glia. 2002; 40:279-91.

11. Tait MJ, Saadoun S, Bell BA, Papadopoulos MC. Water movements in the brain: role of aquaporins. Trends Neurosci. 2008;31:37-43.

12. Fukuda AM, Badaut J. Aquaporin 4: a player in cerebral edema and neuroinflammation. J Neuroinflammation. 2012;9:279.

13. Zhang $R$, Liu Y, Yan $K$, Chen L, Chen XR, Li P, Chen FF, Jiang XD. Antiinflammatory and immunomodulatory mechanisms of mesenchymal stem cell transplantation in experimental traumatic brain injury. J Neuroinflammation. 2013;10:871.

\section{Ready to submit your research? Choose BMC and benefit from:}

- fast, convenient online submission

- thorough peer review by experienced researchers in your field

- rapid publication on acceptance

- support for research data, including large and complex data types

- gold Open Access which fosters wider collaboration and increased citations

- maximum visibility for your research: over 100M website views per year

At BMC, research is always in progress.

Learn more biomedcentral.com/submissions 